\title{
Taurine Improves Lipid Metabolism and Increases Resistance to Oxidative Stress
}

\author{
Zi WANG ${ }^{1}$, Yoshihisa OHATA ${ }^{1}$, Yukari WATANABE ${ }^{1}$, Yiwen YuAN $^{1}$, Yuki YoshiI ${ }^{1}$, \\ Yoshitaka KONDO $^{1}$, Shoko NISHIZONO ${ }^{2}$ and Takuya CHIBA ${ }^{1, *}$ \\ ${ }^{1}$ Biomedical Gerontology Laboratory, Faculty of Human Sciences, Waseda University, \\ Tokorozawa 359-1192, Japan \\ ${ }^{2}$ Department of Applied Microbial Technology, Faculty of Biotechnology and Life Science, \\ Sojo University, Kumamoto 860-0082, Japan
}

(Received November 6, 2019)

\begin{abstract}
Summary Calorie restriction (CR) by 30-40\% decreases morbidity of age-related diseases and prolongs the lifespan of various laboratory animal species. Taurine (2-aminoethanesulfonic acid) is an important nutrient for lipid metabolism as it conjugates bile acids. Here, we investigated how taurine supplementation induces effects similar to the CR beneficial effects. Sprague Dawley rats were fed a diet containing different taurine concentrations $(0$, $0.5,1.0,3.0,5.0 \%)$ to analyze the effects on growth, blood, and hepatic parameters. Rats fed a 5\% taurine-supplemented diet showed a significant decrease in visceral fat weight, compared with control rats. Moreover, there were significant decreases in the serum total cholesterol, hepatic cholesterol and triglyceride concentrations in the taurine-supplemented groups compared with the control group in a dose-dependent manner. These results were associated with decreased mRNA expression of fatty acid synthase, and increased mRNA expression of carnitine palmitoyltransferase $1 \alpha$. C57BL/ 6 mice were fed a $5.0 \%$ taurinesupplemented diet, and their response to 3-nitropropionic acid-induced oxidative stress was analyzed. The rate of weight loss due to oxidative stress decreased and the survival rate significantly increased in the taurine-supplemented groups compared with the control group. Finally, cells were treated with $100 \mu \mathrm{M}$ taurine and their resistance to UV-induced oxidative stress was analyzed. We found that the p53-Chk1 pathway was less activated in taurine-treated cells compared with control cells. Furthermore, damage to cells evaluated by oxidative stress indicators revealed a reduction in oxidative damage with taurine treatment. These findings suggest that taurine partially acts as a CR mimetic.
\end{abstract}

Key Words taurine, oxidative stress, lipid metabolism, calorie restriction, 3-nitropropionic acid, UV

Research on calorie restriction (CR) dates back to a 1935 study by McCay et al., which reported an extension of lifespan in CR rats (1). Since then, it has been established that $30 \%$ to $40 \%$ CR on laboratory animals suppresses the development of age-related diseases and extends their lifespan compared with ad libitum (AL) feeding (2). Furthermore, Colman and colleagues has reported the effects of $30 \% \mathrm{CR}$ in a 20-y study on calorie-restricted rhesus macaques (3). This study found a decrease in the incidence of age-related disorders such as diabetes, cancers, cardiovascular diseases and brain atrophy, as well as a decrease in age-related mortality. These findings demonstrated that $\mathrm{CR}$ in rhesus macaques had a life-extending effect, attributed to the decrease in $\mathrm{t}$ he incidence of age-related diseases $(3,4)$. A National Institute of Aging study on the effects of CR on rhesus macaques was controversial (5), however, it finally noted a healthy life-extending effect similar to the findings of the aforementioned study at University of Wisconsin (6). These results suggest that CR should also be applied on humans. Nonetheless, as it is difficult to

*To whom correspondence should be addressed.

E-mail: takuya@waseda.jp perform long-term CR on humans, CR mimetic compounds, which are chemicals that have anti-aging effects similar to CR without actual food restriction, have been extensively investigated (7-9). We have previously reported that antioxidant effects and adaptation to glucose and lipid metabolism are important at the cellular level (10). Recently, several compounds including taurine (2-aminoethanesulfonic acid), metformin, rapamycin and sirtuin activators were reported to have age-related disease preventive effects similar to $\mathrm{CR}$, and metformin has been used in clinical practice (11). Among these compounds, we focused on taurine, which has been suggested to have effects on lipid metabolism and antioxidative effects that are characteristic of the anti-aging effects of CR. Interestingly, a recent study suggested that taurine has anti-aging effects through its molecular chaperon effect (12).

Taurine, a sulfur-containing amino acid derivative present in high concentrations in various animal tissues, is available from dietary sources and can be synthesized in vivo from other amino acids (13). Taurine is involved in many physiological functions including osmoregulation, antioxidation, calcium modulation and membrane stabilization, which are also associated with 
the prevention of obesity (14). Moreover, analysis of taurine transporter-knockout mice suggests that taurine has a significant role in maintaining normal cellular functions to prevent early onset of aging $(15,16)$. This does not necessarily imply that taurine intake has an anti-aging effect, however, it does suggest that it affects aging and the development of age-related diseases.

Taurine exists in all tissues of the human body and is especially highly concentrated in tissues such as the heart, skeletal muscles, liver, brain and retina (17). Taurine can be supplied to the body through food intake or biosynthesis in the organism from methionine or cysteine (18). It has been reported that taurine intake inhibits the increase in mitochondria reactive oxygen species (ROS), which may lead to enhanced oxidative stress (19). Indeed, it has been shown that taurine could act specifically as a mitochondrial antioxidant (20). Taurine also lowers blood lipid levels and raises ketone bodies levels. Moreover, liver perfusion experiments have revealed that a taurine-induced reduction in hepatic cholesteryl ester accumulation was associated with reduced hepatic secretion of lipids, and was inversely related to enhanced ketone body production and fatty acid oxidation in rats fed a high-cholesterol diet (21).

Despite the diverse effects of taurine, the detailed molecular mechanism of its CR mimetic effects has not been clearly elucidated. Thus, taurine's potential actions as a CR mimetic were analyzed using rats, mice and cultured cells.

\section{MATERIALS AND METHODS}

Animals.

Rats: All animal experiment protocols were approved by the Committee on the Ethics of Animal Experiments of the Waseda University (permission number: 2019A112) and Sojo University (permission number: 2013L-13). Four-week-old male Sprague Dawley (SD) rats weighing around $90 \mathrm{~g}$ were purchased from Kyudo (Kumamoto, Japan). Rats were fed a commercially available solid diet CE-2 (CLEA Japan, Inc., Tokyo, Japan) for a 3-d preliminary feeding period, and then they were divided into five dietary groups: control group, $0.5 \%$ $(\mathrm{w} / \mathrm{w})$ taurine-supplemented group, $1.0 \%(\mathrm{w} / \mathrm{w})$ taurine-supplemented group, 3.0\% (w/w) taurine-supplemented group and $5.0 \%(\mathrm{w} / \mathrm{w})$ taurine-supplemented group $(n=7-8)$. The mean weight of each group was almost equal. The diet was prepared in accordance with the AIN-76 mixture supplemented with $0.5 \%$ cholesterol and $0.125 \%$ sodium cholate (high-cholesterol diet). Taurine was provided by Taisho Pharmaceutical Co., Ltd. (Tokyo, Japan). The rearing period was $2 \mathrm{wk}$ and the rearing temperature was kept at $23 \pm 1^{\circ} \mathrm{C}$, with the light on from 8:00 to 20:00. Feed and water were available AL and were replaced every $2 \mathrm{~d}$. After the end of the rearing period, rats were humanely euthanized by decapitation at a timepoint when the cholesterol synthesis pathway would be activated (between 23:00 and 1:00) without prior fasting, and blood was collected. Serum and liver samples were collected and stored at $-80^{\circ} \mathrm{C}$ until analysis. Mesenteric, testicular, and perirenal adipose tissues were also collected and their weight was measured.

Mice: Male C57BL/6J mice aged 17 to 19 wk were purchased from Charles River Laboratories Japan (Yokohama, Japan). After a 3-d preliminary feeding period, mice were kept in groups of four to five, at $23 \pm 1^{\circ} \mathrm{C}$, with the light period on 7:00 to 19:00. Mice were fed AL a solid diet CRF-1 (Oriental Yeast Co., Ltd., Tokyo, Japan) as the standard diet $(n=17)$. Taurine (FUJIFILM Wako Pure Chemical Corporation, Osaka, Japan) was administered AL in conjunction with CRF-1 (5\% w/w) with tap water $(n=15)$. Mice were observed once daily, and the amount of food intake was measured twice a week. During the 3-nitropropionic acid (3NPA, FUJIFILM Wako) injection experiment, the weight of the mice was measured every day to determine the endpoint conditions as described below.

Cultured cells. COS-1 cells derived from African green monkey kidneys were subcultured at $37^{\circ} \mathrm{C}$ in a $5 \% \mathrm{CO}_{2}$ incubator. COS-1 cells are a fibroblast-like, SV40 large T antigen-immortalized cell line suitable for transfection. MEM $\alpha$ (FUJIFILM Wako) containing 5\% fetal bovine serum (FBS) (Biowest, Funakoshi, Tokyo, Japan) and 1\% antibiotic and antibacterial substances (Nacalai Tesque, Inc., Kyoto, Japan) was used as the culture medium. After $48 \mathrm{~h}$ of subculturing, cells were treated with or without $100 \mu \mathrm{M}$ taurine as necessary.

Analysis of serum parameters. Blood was centrifuged at $830 \times g$ for $15 \mathrm{~min}$ at $4^{\circ} \mathrm{C}$ and the supernatant was collected. The serum triglyceride concentration was measured with Triglyceride E-test Wako (GPO-DAOS method, FUJIFILM Wako), the serum cholesterol concentration was measured with Cholesterol E-test Wako (Cholesterol oxidase-DAOS method, FUJIFILM Wako), and the serum phospholipid concentration was measured with Phospholipid E-test Wako (Choline oxidaseDAOS method, FUJIFILM Wako). High-density lipoprotein (HDL) cholesterol was fractionated (FUJIFILM Wako), and then measured in the same manner as the total cholesterol concentration. The serum glucose concentration was measured using Glucose CII-Test Wako (mutarotase-GOD method, FUJIFILM Wako).

Analysis of hepatic lipids. Hepatic lipids were extracted according to the Folch method (22). In brief, $1.0 \mathrm{~mL}$ of hepatic lipid extracts was divided into two: one for triglyceride measurement and the other for phospholipid measurement, and then frozen until use. Lipid extracts were measured using the same kits as those used for the serum.

Induction of oxidative stress. To examine the response and resistance to oxidative stress in taurine-administrated mice, 3NPA (FUJIFILM Wako) was used. After $88 \mathrm{~d}$ from the start date of the rearing experiment, $3 \mathrm{NPA}[75 \mathrm{mg} / \mathrm{kg}$ body weight (BW)] dissolved in phosphate-buffered saline (PBS) was intraperitoneally (ip) administered without anesthesia twice a week. For controls, the same amount of PBS was administered instead of 3NPA. Mice were divided into the following groups: 1 ) normal diet with nine rounds of ip administration of 
3NPA (AL); 2) 5\% (w/w) taurine-supplemented diet with nine rounds of ip administration of 3NPA (AL+ Tau); 3) normal diet without application of oxidative stress (control, Tau -, 3NPA-); 4) normal diet with two rounds of ip administration of 3NPA (Tau-, 3NPA+); 5) $5 \%(\mathrm{w} / \mathrm{w})$ taurine-supplemented diet with two rounds of ip administration of 3NPA (Tau+, 3NPA+).

The survival rate of mice on normal diet (AL) or $5 \%$ $(\mathrm{w} / \mathrm{w})$ taurine-supplemented $\operatorname{diet}(\mathrm{AL}+\mathrm{Tau})$ that received nine rounds of $3 \mathrm{NPA}$ was examined. Furthermore, their weight loss rate 4 wk after $3 \mathrm{NPA}$ administration was analyzed. For 3NPA-administered mice, the endpoint was set to the point in time when moribund conditions including symptoms such as reduced body temperature, cyanosis, decreased physical activity and $>20 \%$ BW reduction were observed. Whole blood was collected from the heart under $2.5 \%$ isoflurane anesthesia, and then the mice were euthanized. For gene expresion analysis, mice were euthanized $30 \mathrm{~min}$ after the second round of $3 \mathrm{NPA}$ or PBS administration and liver samples were collected.

Measuring gene expression levels. Hepatic gene expression levels were measured according to the manufacturer's protocol using real-time reverse transcription-PCR (real-time RT-PCR) on an ABI Prism 7000 Sequence Detection System (Thermo Fisher Scientific, Waltham, MA, USA). In brief, liver samples were homogenized using a homogenizer, and RNA was collected using RNeasy Plus Universal (Qiagen, Hilden, Germany). Next, cDNA was generated from a set amount of RNA using the High Capacity cDNA Reverse Transcription Kit (Thermo Fisher Scientific). Gene-specific amplification was detected using TaqMan ${ }^{\circledR}$ Universal PCR Master Mix and Assays-on-demand ${ }^{\mathrm{TM}}$ (Thermo Fisher Scientific). Analyzed genes are follows: fatty acid synthase (Fasn), glucose 6-phosphate dehydrogenase (G6pd), carnitine palmitoyltransferase 1 a (Cpt1a), cholesterol $7 \alpha$ hydroxylase (Cyp7a1), peroxisome proliferator-activated receptor $\alpha$ (Ppara), peroxisome proliferator-activated receptor $\gamma$ (Pparg), peroxisome proliferator-activated receptor $\gamma$ coactivator-1 $\alpha$ (Ppargc1a), sterol regulatory element-binding factor 1 (Srebf1), sterol regulatory element-binding factor 2 (Srebf2), liver X receptor $\alpha$ (Lxr), glucokinase (Gck), phosphoenolpyruvate carboxykinase (Pepck), mitochondrial transcription factor A (Tfam), glyceraldehyde-3-phosphate dehydrogenase (Gapdh), sirtuin 3 (Sirt3), ATP-binding cassette transporter b11 (Abcb11), ATP-binding cassette transporter G5 (Abcg5), ATP-binding cassette transporter G8 (Abcg8), glutathione S-transferase mu 3 (Gstm3) and Heme oxygenase 1 (Hmox 1$)$.

Protein extraction. Liver tissues (20-50 mg) were homogenized in $1 \mathrm{~mL}$ of T-PER ${ }^{\circledR}$ Tissue Protein Extraction (Thermo Fisher Scientific) containing 1\% Phosphatase Inhibitor Cocktail 3 (Sigma-Aldrich, St. Louis, MO, USA), Protease Inhibitor Cocktail and animal component-free media (Sigma-Aldrich) on ice using a homogenizer for $30 \mathrm{~s} \times 3$ cycles. Homogenates were then centrifuged for $10 \mathrm{~min}$ at $10,000 \times g$ at $4^{\circ} \mathrm{C}$, and the supernatants were used as protein extracts. Proteins are extracted in the same manner from cultured cells.
Protein concentration was determined using BCA Protein Assay Kit (TaKaRa, Kusatsu, Japan) according to the manufacturer's protocol; absorbance was measured at $560 \mathrm{~nm}$ on a ChroMate ${ }^{\circledR}$ Microplate Reader Model 4300 (Awareness Technology, Palm City, FL, USA). Protein solutions were adjusted to a concentration of 2 $\mathrm{mg} / \mathrm{mL}$ using T-PER. Sample buffer containing $2 \times$ Laemmli Sample Buffer (Bio-Rad, Hercules, CA, USA) and $0.05 \times 2$-mercaptoethanol (Bio-Rad) was then added to an equal volume of sample. The solution was denatured at $100^{\circ} \mathrm{C}$ for $5 \mathrm{~min}$. The sample was then placed on ice for $3 \mathrm{~min}$, spun-down and stored at $-30^{\circ} \mathrm{C}$.

SDS polyacrylamide gel electrophoresis (PAGE) and western blot analysis. Pre-stained Protein Markers (Broad Range) for SDS-PAGE (Nacalai Tesque, Inc., Kyoto, Japan) and Magic Mark ${ }^{\mathrm{TM}}$ XP Western Standard (Thermo Fisher Scientific) were used as molecular weight markers. The markers were denatured at $100^{\circ} \mathrm{C}$ for $5 \mathrm{~min}$ in the same way as the protein samples. TGX ${ }^{\mathrm{TM}}$ FastCast ${ }^{\mathrm{TM}}$ Acrylamide Kit (Bio-Rad), TEMED (Bio-Rad) and Ammonium Persulfate (APS; Bio-Rad) were used to separate the proteins according to the manufacturers' protocols. The gel was placed in a Mini-PROTEAN ${ }^{\circledR}$ Tetra Cell (BioRad), to which $1 \times$ running buffer [Tris/Glycine/SDS Buffer (Bio-Rad)] was added. The protein samples $(10 \mu \mathrm{L}$ each) were added to the wells. The protein samples were electrophoresed at $200 \mathrm{~V}$ and $80 \mathrm{~mA}$ or greater for $30 \mathrm{~min}$. The gel was placed on a Trans-Blot ${ }^{\circledR}$ Turbo $^{\mathrm{TM}}$ Transfer Pack (Bio-Rad) and the voltage was set at $25 \mathrm{~V}$ or less, the current at $1.0 \mathrm{~A}$ and the running time at 30 min according to the manufacturer's protocol, using an Immun-Blot ${ }^{\circledR}$ PVDF Membrane for Protein Blotting (Bio-Rad). After protein transfer, the membrane was rinsed three times for $10 \mathrm{~min}$ with TBS-T [TBS (Bio-Rad) in MilliQ water with $0.1 \%$ Tween 20 (FUJIFILM Wako)]. The membrane was blocked for 20 min with Blocking One (Nacalai) or Blocking One-P (Nacalai).

The primary antibodies were diluted $1: 1,000$ with either Blocking One or Blocking One-P and incubated with agitation with the membrane at $4^{\circ} \mathrm{C}$ overnight. Subsequently, the membrane was rinsed three times for 10 min with TBS-T. The secondary antibodies were diluted 1 : 5,000 with Blocking One and incubated with agitation with the membrane for $1 \mathrm{~h}$ at room temperature. Antibodies used include p-p53 (Ser15) antibody (CST, Danvers, MA, USA), p53 (1C12) antibody (CST), p-chk1 (S345) (133D3) antibody (CST), $\beta$-Tubulin antibody (FUJIFILM Wako), anti-mouse IgG $(\mathrm{H}+\mathrm{L})$ (FUJIFILM Wako), stabilized goat anti-rabbit HRP-conjugated antibody (Thermo Fisher Scientific), anti-rabbit IgG and HRP-linked antibody (CST).

After reacting with the secondary antibodies, the membrane was rinsed three times for 10 min with TBS$\mathrm{T}$ before it was visualized with the SuperSignal ${ }^{\circledR}$ West Femto Maximum Sensitivity Substrate SuperSignal (Thermo Fisher Scientific) according to the manufacturer's protocol. LAS3000 (Fujifilm, Tokyo, Japan) was used to capture images and quantify the band intensity. Stripping Solution (FUJIFILM Wako) was used as necessary to strip an antibody and reprobe with another 
Table 1. Taurine-supplemented diet significantly reduces mesenteric white adipose tissue weight in SD rats.

\begin{tabular}{|c|c|c|c|c|c|}
\hline \multirow{2}{*}{ Groups } & \multirow{2}{*}{ Control } & \multicolumn{4}{|c|}{ Taurine supplementation (\%) } \\
\hline & & $0.5 \%$ & $1.0 \%$ & $3.0 \%$ & $5.0 \%$ \\
\hline Initial body weight (g) & $158 \pm 2$ & $156 \pm 3$ & $156 \pm 2$ & $157 \pm 2$ & $157 \pm 2$ \\
\hline Final body weight (g) & $287 \pm 6$ & $279 \pm 8$ & $280 \pm 5$ & $284 \pm 4$ & $279 \pm 6$ \\
\hline Body weight gain $(\mathrm{g})$ & $129 \pm 4$ & $123 \pm 5$ & $123 \pm 3$ & $127 \pm 4$ & $121 \pm 4$ \\
\hline Food intake $(\mathrm{g} / \mathrm{d})$ & $22.9 \pm 0.7$ & $21.9 \pm 0.7$ & $22.0 \pm 0.6$ & $22.3 \pm 0.5$ & $22.6 \pm 0.6$ \\
\hline Food efficiency (\%) & $40.2 \pm 0.012$ & $40.1 \pm 0.7$ & $40.1 \pm 0.6$ & $40.6 \pm 0.7$ & $41.2 \pm 0.7$ \\
\hline Liver weight (g/100 g body weight) & $5.92 \pm 0.18$ & $5.92 \pm 0.22$ & $5.93 \pm 0.15$ & $5.64 \pm 0.08$ & $5.57 \pm 0.18$ \\
\hline \multicolumn{6}{|l|}{ WAT weight (g/100 g body weight) } \\
\hline Mesenteric & $1.104 \pm 0.059^{\mathrm{a}}$ & $0.955 \pm 0.076^{\mathrm{ab}}$ & $0.917 \pm 0.039^{\mathrm{ab}}$ & $1.033 \pm 0.049^{\mathrm{ab}}$ & $0.871 \pm 0.042^{b}$ \\
\hline Epididymal & $1.056 \pm 0.051$ & $1.064 \pm 0.056$ & $1.038 \pm 0.038$ & $1.098 \pm 0.069$ & $0.989 \pm 0.057$ \\
\hline Perirenal+retroperitoneal & $1.457 \pm 0.113$ & $1.279 \pm 0.149$ & $1.341 \pm 0.135$ & $1.315 \pm 0.1$ & $1.212 \pm 0.079$ \\
\hline Total & $3.617 \pm 0.182$ & $3.298 \pm 0.267$ & $3.269 \pm 0.159$ & $3.445 \pm 0.181$ & $3.072 \pm 0.13$ \\
\hline
\end{tabular}

Mean values \pm SE of physical parameters are shown $(n=6-7)$. Different superscript letters indicate significant differences $(p<0.05)$.

WAT: white adipose tissue.

Table 2. Taurine-supplemented diet improves the blood cholesterol levels in SD rats.

\begin{tabular}{lccccc}
\hline \multirow{2}{*}{\multicolumn{1}{c}{ Groups }} & Control & \multicolumn{3}{c}{ Taurine supplementation (\%) } \\
\cline { 3 - 6 } & & $0.5 \%$ & $1.0 \%$ & $3.0 \%$ & $5.0 \%$ \\
\hline Total cholesterol (mg/dL) & $222 \pm 16^{\mathrm{a}}$ & $154 \pm 19^{\mathrm{b}}$ & $142 \pm 10^{\mathrm{b}}$ & $141 \pm 14^{\mathrm{b}}$ & $125 \pm 10^{\mathrm{b}}$ \\
HDL-cholestrol (mg/dL) & $27.1 \pm 4.2$ & $29.7 \pm 1.1$ & $30.3 \pm 2.5$ & $27.7 \pm 2.2$ & $32.6 \pm 3.2$ \\
HDL-CHOL/T-CHOL (\%) & $12.5 \pm 1.9^{\mathrm{b}}$ & $19.6 \pm 3.3^{\mathrm{ab}}$ & $22.7 \pm 3.2^{\mathrm{ab}}$ & $20.8 \pm 2.4^{\mathrm{ab}}$ & $26.6 \pm 2.8^{\mathrm{a}}$ \\
Triglyceride (mg/dL) & $120 \pm 23$ & $117 \pm 24$ & $124 \pm 12$ & $105 \pm 17$ & $112 \pm 6$ \\
Phospholipids (mg/dL) & $157 \pm 10$ & $158 \pm 13$ & $150 \pm 5$ & $131 \pm 6$ & $142 \pm 9$ \\
Glucose (mg/dL) & $169 \pm 7$ & $170 \pm 6$ & $176 \pm 6$ & $164 \pm 7$ & $170 \pm 2$ \\
\hline
\end{tabular}

Mean values \pm SE of blood parameters in rats are shown $(n=6-7)$. Different superscript letters indicate significant differences $(p<0.05)$.

HDL-CHOL: high density lipoprotein-cholesterol, T-CHOL: total cholesterol.

antibody.

Taurine treatment and UV radiation. D-PBS (FUJIFILM Wako) was used to prepare a $100 \mathrm{~mm}$ Taurine (FUJIFILM Wako) stock solution. This taurine solution was filtered through a 0.22- $\mu \mathrm{m}$ Millipore LCR (Hydrophilic PTFE) Membrane (MILLIPRE, Danvers, MA, USA) for sterilization. Taurine was diluted in MEM $\alpha$ to a final concentration of $100 \mu \mathrm{M}$. This medium was used for preprocessing before UV radiation and for cultivation after UV radiation for a certain amount of time. Cells were washed with D-PBS before UV radiation and CL1000 Ultraviolet Crosslinker (UVP, NH, USA) was used to apply $120 \mathrm{~mJ} / \mathrm{cm}^{2}$ of UV radiation on cells. The distance of light to cells was $12.5 \mathrm{~cm}$, and the approximate irradiation period was $20 \mathrm{~s}$. The main wavelength of UV light was $302 \mathrm{~nm}$ (UVB).

Detection of oxidative stress. To analyze the protective effect of taurine against UV treatment, an experiment was conducted using an oxidative stress indicator. Six hours after UV treatment, cells were dissociated with $0.25 \%$ trypsin EDTA, which was inactivated with MEM $\alpha$ containing 5\% FBS. The supernatants were discarded after centrifugation and $\mathrm{CM}-\mathrm{H}_{2}$ DCFDA (Thermo Fisher
Scientific) and MEM $\alpha$ containing 5\% FBS were added for incubation at $37^{\circ} \mathrm{C}$ and $5 \% \mathrm{CO}_{2}$ in air for $30 \mathrm{~min}$. Green fluorescence derived from ROS was detected using a BZ9000 fluorescence microscope (Keyence, Osaka, Japan). Moreover, to study the mitochondria protective function of taurine against UV treatment, cells were incubated with medium containing $10 \mu \mathrm{M} \mathrm{JC}-1$ (Cayman Chemical Company, Ann Arbor, MI, USA) at $37^{\circ} \mathrm{C}$ and $5 \%$ $\mathrm{CO}_{2}$ in air for $30 \mathrm{~min}$. The cells were then washed with D-PBS to remove the JC- 1 , and then placed in MEM $\alpha$ containing 5\% FBS. Finally, red fluorescence and green fluorescence derived from mitochondrial membrane potential was detected using a BZ-9000 fluorescence microscope.

Statistical analysis. The data are presented as the mean values $\pm S E$ (experiment with rats) or mean values \pm SD (experiment with mice or cells). The data were analyzed by one-way ANOVA and $t$-test, using IBM SPSS Statistic 22 and Microsoft Office Excel 2007. The KaplanMeier method and the log rank test were used to analyze the survival rates. The Tukey-Kramer method was used for post-hoc tests. Moreover, for parameters that appeared to be affected by taurine intake, simple linear 
Table 3. Taurine-supplemented diet lowers the hepatic cholesterol and triglyceride levels in SD rats.

\begin{tabular}{lccccc}
\hline \multirow{2}{*}{ Groups } & Control & \multicolumn{4}{c}{ Taurine supplementation (\%) } \\
\cline { 3 - 6 } & & $0.5 \%$ & $1.0 \%$ & $3.0 \%$ & $5.0 \%$ \\
\hline Total cholesterol (mg/g liver) & $72.1 \pm 3.5^{\mathrm{a}}$ & $59.9 \pm 4.9^{\mathrm{ab}}$ & $64.4 \pm 4.9^{\mathrm{ab}}$ & $51.7 \pm 3.3^{\mathrm{b}}$ & $49.9 \pm 2.8^{\mathrm{b}}$ \\
Triglyceride (mg/g liver) & $109.7 \pm 9.0^{\mathrm{a}}$ & $94.8 \pm 7.3^{\mathrm{ab}}$ & $74.8 \pm 5.2^{\mathrm{b}}$ & $81.4 \pm 7.6^{\mathrm{ab}}$ & $79.0 \pm 6.5^{\mathrm{b}}$ \\
Phospholipids (mg/g liver) & $31.4 \pm 5.7$ & $23.0 \pm 0.6$ & $23.0 \pm 0.9$ & $24.5 \pm 2.4$ & $28.5 \pm 2.2$ \\
\hline
\end{tabular}

Mean values \pm SE of hepatic parameters are shown $(n=6-7)$. Different superscript letters indicate significant differences $(p<0.05)$.

Table 4. Taurine-supplemented diet regulates the hepatic expression of genes associated with lipid metabolism in SD rats.

\begin{tabular}{|c|c|c|c|c|c|c|}
\hline \multirow{2}{*}{ Groups } & \multirow{2}{*}{ Control } & \multicolumn{4}{|c|}{ Taurine supplementation (\%) } & \multirow{2}{*}{$R$} \\
\hline & & $0.5 \%$ & $1.0 \%$ & $3.0 \%$ & $5.0 \%$ & \\
\hline Fasn & $1.00 \pm 0.31$ & $1.14 \pm 0.29$ & $0.57 \pm 0.16$ & $0.35 \pm 0.05$ & $0.42 \pm 0.07$ & $-0.40^{*}$ \\
\hline G6pd & $1.00 \pm 0.18$ & $1.09 \pm 0.16$ & $1.20 \pm 0.25$ & $1.13 \pm 0.24$ & $0.68 \pm 0.09$ & -0.24 \\
\hline Cpt1a & $1.00 \pm 0.07^{\mathrm{ab}}$ & $0.91 \pm 0.12^{\mathrm{b}}$ & $1.45 \pm 0.33^{\mathrm{ab}}$ & $1.85 \pm 0.35^{\mathrm{ab}}$ & $1.92 \pm 0.25^{\mathrm{a}}$ & $0.52^{*}$ \\
\hline Сур7a1 & $1.00 \pm 0.19^{\mathrm{ab}}$ & $1.32 \pm 0.17^{\mathrm{a}}$ & $0.68 \pm 0.18^{b}$ & $0.35 \pm 0.09^{b}$ & $0.42 \pm 0.15^{b}$ & $-0.53^{*}$ \\
\hline Ppara & $1.00 \pm 0.30$ & $1.22 \pm 0.31$ & $0.35 \pm 0.09$ & $1.07 \pm 0.30$ & $0.47 \pm 0.11$ & -0.22 \\
\hline Pparg & $1.00 \pm 0.22^{\mathrm{a}}$ & $0.97 \pm 0.22^{\mathrm{ab}}$ & $0.50 \pm 0.20^{\mathrm{abc}}$ & $0.25 \pm 0.08^{c}$ & $0.16 \pm 0.07^{\mathrm{c}}$ & $-0.55^{*}$ \\
\hline Ppargc1a & $1.00 \pm 0.22^{\mathrm{a}}$ & $0.82 \pm 0.12^{\mathrm{ab}}$ & $0.57 \pm 0.15^{\mathrm{ab}}$ & $0.29 \pm 0.05^{b}$ & $0.33 \pm 0.05^{\mathrm{b}}$ & $-0.55^{*}$ \\
\hline Srebf1 & $1.00 \pm 0.12^{\mathrm{a}}$ & $0.87 \pm 0.11^{\mathrm{ab}}$ & $0.60 \pm 0.19^{\mathrm{abc}}$ & $0.42 \pm 0.08^{b c}$ & $0.35 \pm 0.10^{c}$ & $-0.58^{*}$ \\
\hline Srebf 2 & $1.00 \pm 0.14^{\mathrm{ab}}$ & $1.09 \pm 0.14^{\mathrm{a}}$ & $0.71 \pm 0.22^{\mathrm{ab}}$ & $0.46 \pm 0.08^{b}$ & $0.44 \pm 0.12^{\mathrm{b}}$ & $-0.51^{*}$ \\
\hline$L x r$ & $1.00 \pm 0.12^{\mathrm{ab}}$ & $1.23 \pm 0.18^{\mathrm{a}}$ & $0.65 \pm 0.19^{b c}$ & $0.37 \pm 0.06^{\mathrm{c}}$ & $0.33 \pm 0.07^{c}$ & $-0.51^{*}$ \\
\hline Gck & $1.00 \pm 0.24^{\mathrm{a}}$ & $0.72 \pm 0.12^{\mathrm{a}}$ & $0.32 \pm 0.09^{\mathrm{a}}$ & $0.18 \pm 0.03^{b}$ & $0.20 \pm 0.05^{\mathrm{b}}$ & $-0.57^{*}$ \\
\hline Pepck & $1.00 \pm 0.25$ & $1.56 \pm 0.33$ & $1.15 \pm 0.31$ & $1.04 \pm 0.23$ & $0.64 \pm 0.12$ & 0.30 \\
\hline Tfam & $1.00 \pm 0.31^{\mathrm{a}}$ & $0.90 \pm 0.26^{\mathrm{ab}}$ & $0.38 \pm 0.15^{\mathrm{ab}}$ & $0.19 \pm 0.04^{b}$ & $0.12 \pm 0.03^{b}$ & $-0.52^{*}$ \\
\hline Gapdh & $1.00 \pm 0.24^{\mathrm{ab}}$ & $1.01 \pm 0.17^{\mathrm{a}}$ & $0.74 \pm 0.28^{\mathrm{ab}}$ & $0.38 \pm 0.08^{\mathrm{ab}}$ & $0.22 \pm 0.06^{b}$ & $-0.53^{*}$ \\
\hline Sirt3 & $1.00 \pm 0.11$ & $1.10 \pm 0.20$ & $0.68 \pm 0.14$ & $0.59 \pm 0.14$ & $0.55 \pm 0.08$ & $-0.42^{*}$ \\
\hline$A b c b 11$ & $1.00 \pm 0.18^{\mathrm{ab}}$ & $1.33 \pm 0.16^{\mathrm{a}}$ & $0.61 \pm 0.15^{\mathrm{bc}}$ & $0.31 \pm 0.08^{c}$ & $0.44 \pm 0.09^{b c}$ & $-0.54^{*}$ \\
\hline $\operatorname{Abcg} 5$ & $1.00 \pm 0.18^{\mathrm{ab}}$ & $1.35 \pm 0.24^{\mathrm{a}}$ & $0.73 \pm 0.22^{\mathrm{ab}}$ & $0.49 \pm 0.08^{b}$ & $0.33 \pm 0.08^{b}$ & $-0.53^{*}$ \\
\hline$A b c g 8$ & $1.00 \pm 0.20$ & $0.92 \pm 0.23$ & $0.41 \pm 0.13$ & $0.46 \pm 0.10$ & $0.38 \pm 0.14$ & $-0.41^{*}$ \\
\hline
\end{tabular}

Mean values \pm SE of relative gene expression and the correlation between taurine supplementation and gene expression are shown $(n=6-7)$.

Different superscript letters indicate significant differences $(p<0.05)$.

$R$ : correlation coefficient between relative gene expression and taurine supplementation. ${ }^{*} p<0.05$.

Fasn: fatty acid synthase, G6pd: glucose 6-phosphate dehydrogenase, Cpt1a: carnitine palmitoyltransferase 1a, Cyp7a1: cholesterol $7 \alpha$-hydroxylase, Ppara: peroxisome proliferator-activated receptor $\alpha$, Pparg: peroxisome proliferator-activated receptor $\gamma$, Ppargc1a: peroxisome proliferator-activated receptor $\gamma$ coactivator- $1 \alpha$, Srebf1: sterol regulatory element-binding factor 1, Srebf2: sterol regulatory element-binding factor 2, Lxr: liver X receptor $\alpha$, Gck: glucokinase, Pepck: phosphoenolpyruvate carboxykinase, Tfam: mitochondrial transcription factor A, Gapdh: glyceraldehyde-3-phosphate dehydrogenase, Sirt3: sirtuin 3, Abcb11: ATP-binding cassette transporter b11, Abcg5: ATP-binding cassette transporter G5, and Abcg8: ATP-binding cassette transporter G8.

regression analysis was performed between the parameter and the taurine dose. $p<0.05$ was deemed statistically significant. Parameters that demonstrated significant differences in the $t$-test were marked with an asterisk $\left(^{*}\right)$ and those that demonstrated significant differences in the Tukey-Kramer are shown with different alphabets.

\section{RESULTS}

Taurine-supplemented diet reduces the mesenteric white adipose tissue weight in SD rats

Rats were fed taurine-supplemented diets with differ- ent taurine concentrations $(0.5-5 \%)$, and their basic physical parameters were measured and shown in Table 1. In all groups, no differences were noted in the initial weight, final weight, weight gain, mean intake level, or food efficiency. Moreover, no differences were noted in the liver weight per $100 \mathrm{~g}$ of BW among the groups. White adipose tissues were dissected from the mesentery, epididymis, and perirenal space and their weight was measured. The mesenteric white adipose tissue weight per $100 \mathrm{~g}$ of BW was significantly decreased in the $5.0 \%$ taurine-supplemented group, compared with the control group. 

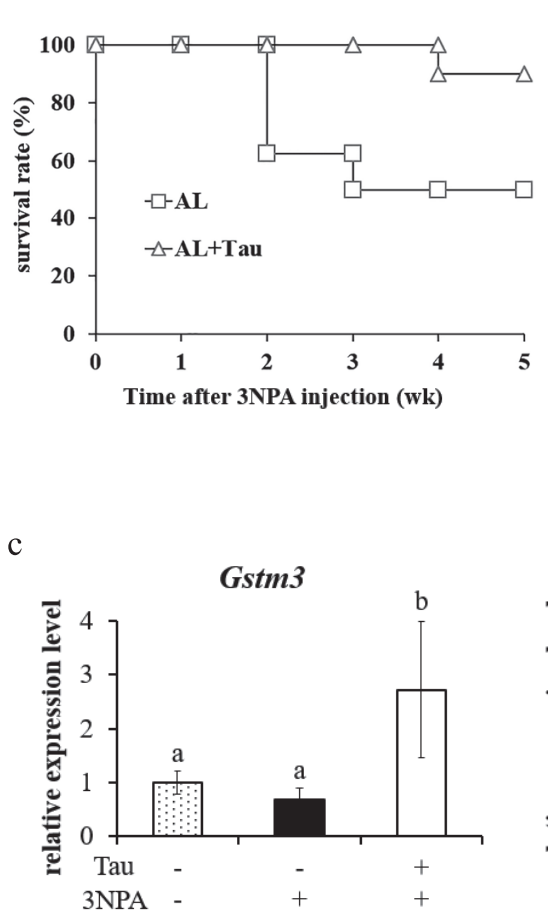
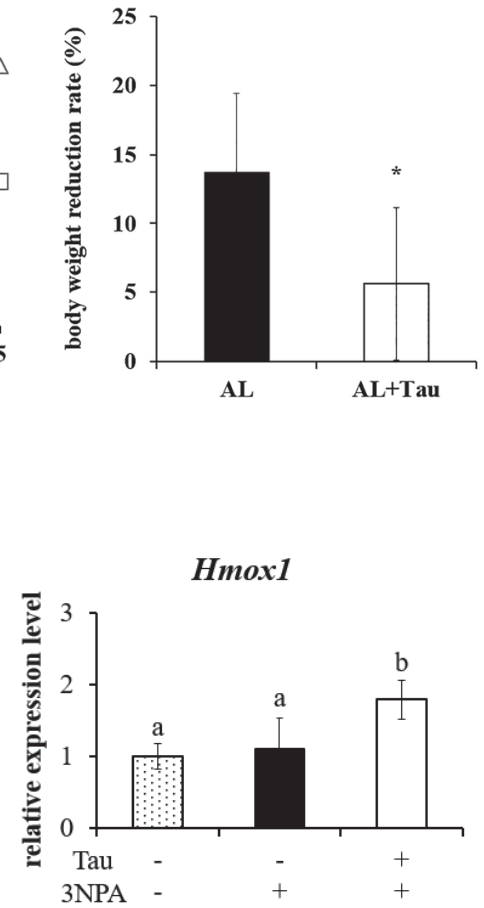

Fig. 1. Taurine-supplemented diet improves resistance to oxidative stress in mice. (a) The survival rate of mice after 3NPA administration. $\square$ represents the survival curve of mice fed a normal diet $(n=8)$, and $\triangle$ represents the survival curve of mice fed a taurine-supplemented diet $(n=10)$. (b) Weight loss rate $4 \mathrm{wk}$ after 3NPA administration $(n=4-9) .{ }^{*} p<0.05$. (c) Gene expression analysis of liver samples by real-time PCR $(n=4-5)$. Different superscript letters indicate significant differences by one-way ANOVA $(p<0.05)$. 3NPA: 3-nitropropionic acid, Gstm3: glutathione $S$-transferase mu 3, and Hmox 1: heme oxygenase 1. Mean values \pm SD are shown.

Taurine-supplemented diet improves the blood cholesterol levels in SD rats

Serum lipid and glucose levels are shown in Table 2. In line with previous reports (21), the total cholesterol concentration was lower in the taurine-supplemented diet groups, including the $0.5 \%$ taurine-supplemented group, compared with the control group. A simple linear regression analysis between the total cholesterol level and the taurine dose revealed a significant inverse correlation $(R=-0.407, p<0.05$; Supplemental Online Material, Fig. S1).

No differences were noted in the HDL-cholesterol level between any of the groups. The ratio of the HDL-cholesterol level to the total cholesterol level was significantly higher only in the $5.0 \%$ group compared with the control group. No differences between the groups were found in the serum triglyceride concentration, phospholipid level, or serum glucose level.

Taurine-supplemented diet improves hepatic cholesterol and triglyceride levels in $S D$ rats

Table 3 shows the measurements of hepatic lipid levels in taurine-supplemented diet fed SD rats. In line with previous reports (21), taurine intake induced a dosedependent decrease in the hepatic cholesterol level, which was significantly lower in the 3.0\% and 5.0\% taurine-supplemented groups compared with the control group. Moreover, taurine intake also induced a dosedependent decrease in the hepatic triglyceride level, which was significantly lower in the $1.0 \%$ and $5.0 \%$ taurine-supplemented groups compared with the control group. There were no differences in the levels of hepatic phospholipids. Simple linear regression analyses between the taurine dose and the hepatic cholesterol level or neutral lipid level demonstrated a significant inverse correlation in both the hepatic cholesterol level $(R=-0.537, p<0.05)$ and the hepatic triglyceride level $(R=-0.346, p<0.05$; Supplemental Online Material, Fig. S2).

Taurine-supplemented diet changes the expression levels of lipid metabolism-related genes

To analyze the taurine-induced lipid metabolism regulatory mechanism, the mRNA expression levels of lipid metabolism-related genes in the liver were measured. As shown in Table 4, the expression level of Fasn was reduced by taurine in a dose dependent manner, and a significant inverse correlation $(R=-0.403, p<0.05)$ was observed. No differences were observed in the expression level of G6pd, which is involved in the supply of NADPH. The expression level of Cpt1a, which is involved in $\beta$-oxidation of fatty acids, showed an increasing tendency with taurine intake in a dose-dependent manner and a positive correlation $(R=0.518, p<0.05)$ was observed. The expression level of Cyp7a1, which is involved in the biosynthesis of bile acids from cholesterol, showed a decreasing trend with taurine intake in a dose-dependent manner. Additionally, the expression levels of Pparg, Ppargc1a, Srebf1, Srebf2 and Lxr, decreased with taurine intake in a dose-dependent man- 
a

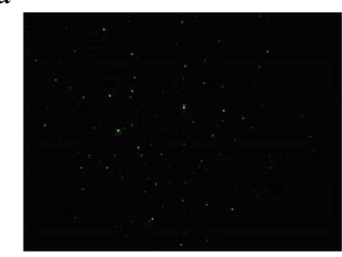

Tau(-) UV(-)

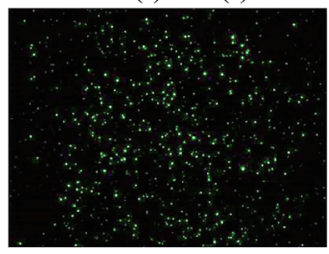

$\operatorname{Tau}(-) \mathrm{UV}(+)$

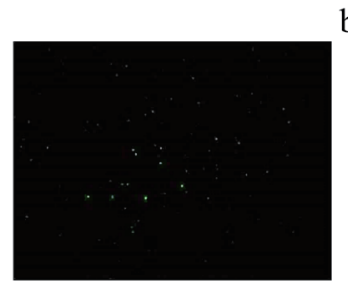

$\operatorname{Tau}(+) \mathrm{UV}(-)$

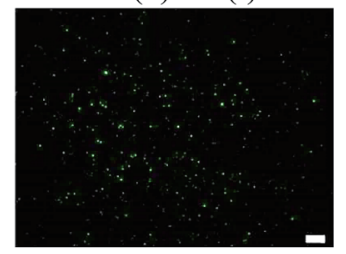

$\operatorname{Tau}(+) \mathrm{UV}(+)$

$\mathrm{b}$
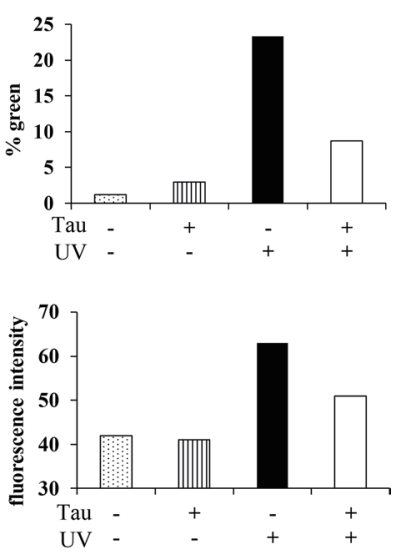

d
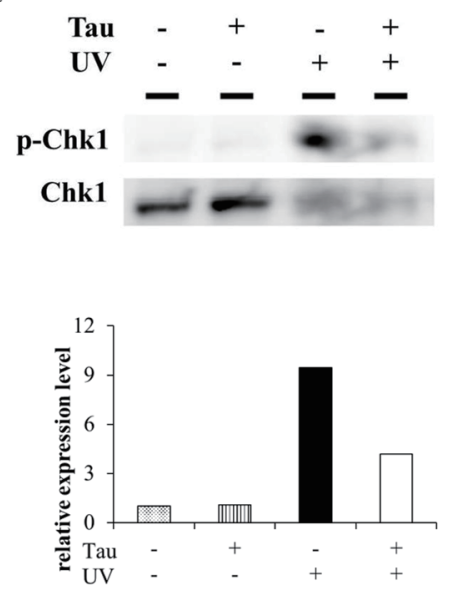

Fig. 2. Taurine supplementation improves resistance to oxidative stress in cultured cells. (a) The analysis was carried out on COS- 1 cells treated with or without taurine in the presence or absence of UV radiation, which induces oxidative stress [Tau (-) UV (-), Tau (+) UV (-), Tau (-) UV (+), Tau (+) UV (+)]. In the Tau (+) UV (+) sample, the intensity of fluorescence was attenuated compared with the Tau (-) UV (+) sample. Bar: $200 \mu \mathrm{m}$. (b) Top: quantification of the proportion of cells in which DCFDA green fluorescence was observed in (a). Bottom: quantification of the mean fluorescence intensity of cells that emitted green fluorescence. (c) Western blotting of the phosphorylation status of p53. The expression of p-p53 is shown. (d) Western blotting of the phosphorylation status of Chk1. The expression of p-Chk1 is shown. These results are the representative of at least three independent experiments. Representative data from a single culture dish are shown in images and graphs.

ner. However, taurine intake had no effects on the expression level of Ppara. While taurine intake had no effects on Pepck, which is involved in gluconeogenesis, the expression levels of Gck and Tfam, which are involved in mitochondrial functions, decreased with taurine intake in a dose-dependent manner. Similarly, the expression levels of ATP-binding cassette transporters (Abcb11, $A b c g 5$ and $A b c g 8)$, which are involved in the secretion of bile acids and cholesterol excretion, decreased with taurine intake in a dose-dependent manner.

Taurine-supplemented diet enhances resistance to oxidative stress in mice

Mice were fed a 5.0\% taurine-supplemented diet and their resistance to 3NPA-induced oxidative stress as well as change in the gene expression due to the stress response was analyzed. In Fig. 1a, $\square$ represents the survival curve of normal-diet-fed mice (AL, $n=8)$ and D represents the survival curve of taurine-supplementeddiet-fed mice (AL+Tau, $n=10)$. In the normal diet group, some mice were found dead 2 wk after 3NPA ad- ministration, whereas in the taurine-supplemented diet group, no mice were found dead 4 wk after $3 \mathrm{NPA}$ administration. Analysis of the survival rate $5 \mathrm{wk}$ after 3NPA administration using the log-rank test revealed that taurine-supplemented mice had a significantly higher survival rate compared with the normal-diet-fed mice (Fig. 1a). Moreover, a comparison of the weight reduction rates $4 \mathrm{wk}$ after $3 \mathrm{NPA}$ administration revealed that mice fed a taurine-supplemented diet had a significantly reduced weight loss rate compared with mice fed a normal diet (Fig. 1b) $(n=4-9)$. Gstm 3 is a component of GST and a Phase II detoxifying enzyme. Hmox 1 catalyzes heme degradation, producing strong antioxidant biliverdin. The gene expression analysis of liver-derived cDNA samples using real-time PCR demonstrated that the relative expression of Gstm 3 and Hmox 1 was significantly elevated in the group fed a taurine-supplemented diet with 3NPA administration compared with the group fed a normal diet with or without 3NPA administration (Fig. 1c) $(n=4-5)$. 
a

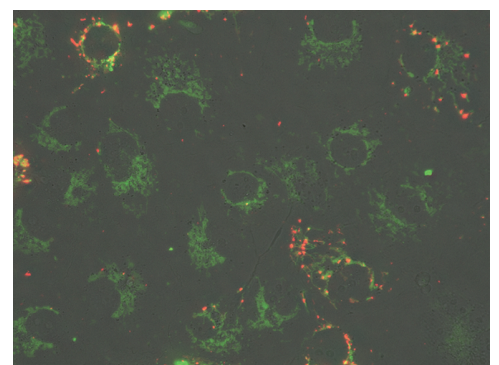

Tau (-) UV (+)

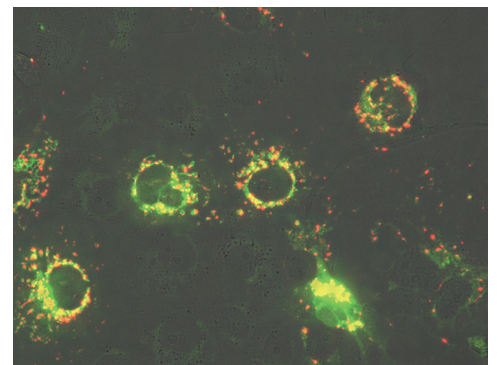

Tau $(+) \mathrm{UV}(+)$ $\mathrm{b}$

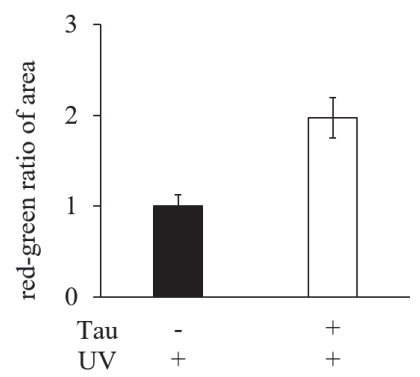

$\mathrm{c}$

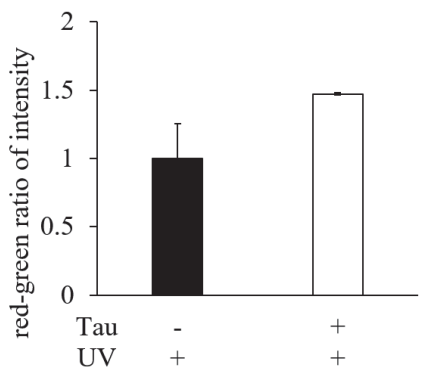

Fig. 3. The analysis of mitochondrial membrane potential through JC-1 assay. (a) Merged red and green fluorescence image from JC-1 assay were shown. Oxidative stress was induced by UV without (left) or with (right) taurine treatment. Bar: $10 \mu \mathrm{m}$. (b) The ratio of red and green fluorescence area measured from (a) were shown. (c) The ratio of red and green fluorescence intensity measured from (a) were shown. These results are the representative of at least three independent experiments.

Taurine suppresses the generation of ROS in cultured cells and maintains mitochondrial functions

DCFDA, a reagent oxidized by ROS to yield fluorescence, was used to analyze the antioxidant effect of taurine. In this experiment, COS-1 cells were treated with or without taurine and subjected or not to UV radiation, which triggers oxidative stress [Tau $(-) \operatorname{UV}(-)$, Tau $(+)$ UV (-), Tau (-) UV (+), Tau (+) UV (+)]. In this experiment, almost no green fluorescence from DCFDA was observed in Tau (-) UV (-) and Tau $(+)$ UV ( -$)$ cells. In contrast, strong green fluorescence was observed after UV radiation in the sample without taurine treatment, Tau (-) UV (+), however, the fluorescence intensity decreased in the sample with taurine treatment, Tau $(+)$ UV $(+)$ (Fig. 2a). Figure 2b shows the quantification of cells in which DCFDA green fluorescence was observed (top panel) and the mean fluorescence intensity of cells that emitted green fluorescence (bottom panel). A decrease in the fluorescence intensity in the Tau $(+)$ UV $(+)$ sample compared with the Tau $(-)$ UV $(+)$ sample was also demonstrated (Fig. 2b).

To confirm the oxidative stress state at the protein level, the protein expression of p53 and Chk1 was analyzed. Although both p53 and Chk1 were phosphorylated upon UV radiation, the level of protein phosphorylation was attenuated in the Tau $(+) \mathrm{UV}(+)$ sample compared with the Tau (-) UV (+) sample (Fig. 2c, d).

We also performed the JC-1 assay to analyze the oxidative stress-induced damage in mitochondria (Fig. 3a). JC-1 dye is a mitochondrial membrane potential indica- tor, which shows red fluorescence in healthy cells with a normal mitochondrial membrane potential. By contrast, in unhealthy or apoptotic cells, JC-1 dye shows its original green fluorescence because of the increased membrane permeability. Thus, healthy cells show a higher red : green fluorescence ratio by JC-1 staining. An increasing trend was observed in the red-green fluorescence ratio in the Tau $(+) \mathrm{UV}(+)$ sample compared with the Tau $(-) \mathrm{UV}(+)$ sample measured by area and intensity using microscopy (Fig. 3b, c). This result indicated that in the Tau (+) UV (+) sample, the mitochondrial membrane potential was sustained compared with the Tau (-) UV (+) sample, even after UV radiation.

\section{DISCUSSION}

Our study showed that 5\% taurine intake significantly reduced the weight of mesenteric white adipose tissue, which corresponds to visceral fat. No significant differences were noted in the body weight or food intake volume between the groups, suggesting there was no growth retardation. In addition, $5 \%$ taurine intake significantly reduced the serum total cholesterol level and increased the ratio of HDL-cholesterol to total cholesterol, which is beneficial for prevention of cardiovascular diseases.

Likewise, the hepatic cholesterol and triglyceride levels were significantly reduced in the $5.0 \%$ taurine-supplemented diet group compared with the control group. Because there was no significant difference in the phospholipid content, these results imply a decrease in cho- 
lesterol and triglyceride levels per cell, rather than a decrease in the number of hepatic cells. These changes in serum and hepatic lipid contents are similar to those seen under CR (10). The expression level of Abcb11, which is involved in the secretion of bile acids, decreased with taurine intake in a dose-dependent manner. Similarly, $A b c g 5$ and $A b c g 8$, which are both involved in the cholesterol flux from the liver, decreased with taurine intake in a dose-dependent manner. Taken together, these results implied that taurine reduced cholesterol and triglyceride levels in the liver by suppressing fatty acid and cholesterol synthesis, rather than by promoting cholesterol flux from the liver. SREBP-1c has been shown to play an important role in the anti-aging effect of CR $(23,24)$. It has been suggested that the diurnal variation in this expression alters the storage and degradation status of lipid acids, which is essential for the anti-aging effect of CR. While previous studies have reported that the cholesterol-reducing effect of taurine partially involved the promotion of bile acids' secretion (21), the present findings indicated a decrease in the expression level of $A b c b 11$. It is possible that this is due to feedback inhibition resulting from the taurine-induced decrease in the hepatic cholesterol level.

The analysis of the survival rate of mice revealed that resistance to 3NPA-induced oxidative stress was significantly improved in mice fed a 5\% taurine-supplemented diet compared with mice fed a normal diet. Likewise, the graph of weight loss rate $4 \mathrm{wk}$ after the start of taurine administration indicated that dietary taurine supplementation improved stress resistance induced by 3NPA. This suggests that taurine administration has a similar improvement effect on the resistance to oxidative stress as that of $\mathrm{CR}$ on mice $(10,25)$. The gene expression analysis showed an elevated expression of Gstm 3 and Hmox1. As we reported previously, both Gstm 3 and Hmox 1 are associated with CR-induced longevity and antioxidant stress resistance (26). Our current study suggests an association between the elevated expression of these genes and taurine administration when oxidative stress was applied, thus improving resistance to oxidative stress. It has been suggested that Nrf2, the transcription factor that regulates the expression of these genes, is activated upon taurine administration (27). Hence, the Nrf2 pathway would be a target of the taurine-induced antioxidative effects.

The experiment using $\mathrm{CM}-\mathrm{H}_{2}$-DCFDA in cultured cells suggested that the intracellular generation of ROS was suppressed by taurine supplementation. In addition, the JC-1 assay revealed that taurine suppressed the reduction in the mitochondrial membrane potential and maintained red fluorescence, suggesting that it suppressed the oxidative stress-induced reduction in the mitochondrial activity. These results suggest that taurine has a protective effect on oxidative stress-induced mitochondrial toxicity, as described in a neurodegenerative model (28). Moreover, the overproduction of ROS from the respiratory chain in hearts of taurine transporter knockout (TauTKO) mice induced mitochondriadependent apoptosis (20). That study also reported a
$60 \%$ decrease in the mitochondrial taurine content of young TauTKO hearts. Furthermore, they demonstrated that treatment of young TauTKO mice with a mitochondria-specific antioxidant, MitoTEMPO, ameliorated activation of the apoptotic pathway in their hearts. Although the precise mechanisms related to how taurine supplementation protected mitochondrial membrane damage in our study are unclear, we speculate that taurine-induced Nrf2-target gene activation is involved in this protective effect. Moreover, taurine supplementation might increase mitochondrial taurine content to suppress oxidative stress-induced apoptosis caused by mitochondrial membrane damage.

Taurine administration inhibited the induction of p53 and Chk1 phosphorylation induced by UV radiation. These findings suggest that taurine reduced UVderived DNA damage and oxidative stress, which induce tumorigenesis (29). Reduction in the phosphorylation of p53 and Chk1 suggests that the UV-induced oxidative stress in the cell was ameliorated by taurine supplementation. However, taurine does not directly inhibit p53 phosphorylation induced by UV radiation. Rather, UV radiation oxidizes DNA, which activates a kinase that phosphorylates p53. Taurine reduces oxidative stress, thereby diminishing DNA damage and decrease p53 and Chk1 phosphorylation. Therefore, taurine might protect mitochondria and genomic DNA against oxidative stress.

Taken together, this study showed that taurine administration in rats improved lipid consumption, suggesting the possibility that lipids dominate as an energy consumption source. This is similar to the changes in metabolism observed in our previous study using CR animals. Moreover, elevated resistance to oxidative stress, which is seen in CR animals, was noted both in vivo and in vitro upon taurine administration. Therefore, our results suggest that taurine administration has CR-like effects by partially exerting similar effects on lipid metabolism and oxidative stress resistance. However, further research is needed to confirm this concept.

\section{Authorship}

Research conception and design: SN and TC; experiments: ZW, YO, YW, YY and YY; statistical analysis of the data: ZW, SN and TC; interpretation of the data: YK, SN and TC.

ZW and YO contributed equally to this work.

\section{Disclosure of state of COI}

The authors declare no conflict of interest.

\section{Acknowledgments}

We are grateful to Shigeru Murakami (Fukui Prefectural University) for helpful discussion. We also thank Maho Kumagai and Kasu Dong for their excellent technical assistance. We thank Michal Bell, PhD, from Edanz Group (www.edanzediting.com/ac) for editing a draft of this manuscript. 


\section{Funding}

This study was supported by Grants-in-Aid for Scientific Research from the Japan Society for the Promotion of Science (TC, nos. 24659181 and 25282027).

\section{Supporting information}

Supplemental online material is available on J-STAGE.

\section{REFERENCES}

1) McCay CM, Crowell MF, Maynard LA. 1935. The effect of retarded growth upon the length of life span and upon the ultimate body size. Nutrition 10: 63-79.

2) Weindruch R, Wolford RL. 1988. The Retardation of Aging and Disease by Dietary Restriction. Charles C Thomas Publisher, Springfield.

3) Colman RJ, Anderson RM, Johnson SC, Kastman EK, Kosmatka KJ, Beasley TM, Allison DB, Cruzen C, Simmons HA, Kemnitz JW, Weindruch R. 2009. Caloric restriction delays disease onset and mortality in rhesus monkeys. Science 325: 201-204.

4) Colman RJ, Beasley TM, Kemnitz JW, Johnson SC, Weindruch R, Anderson RM. 2014. Caloric restriction reduces age-related and all-cause mortality in rhesus monkeys. Nature Commun 5: 3557.

5) Mattison JA, Roth GS, Beasley TM, Tilmont EM, Handy AM, Herbert RL, Longo DL, Allison DB, Young JE, Bryant M, Barnard D, Ward WF, Qi W, Ingram DK, de Cabo R. 2012. Impact of caloric restriction on health and survival in rhesus monkeys from the NIA study. Nature 489: 318-321.

6) Mattison JA, Colman RJ, Beasley TM, Allison DB, Kemnitz JW, Roth GS, Ingram DK, Weindruch R, de Cabo R, Anderson RM. 2017. Caloric restriction improves health and survival of rhesus monkeys. Nature Commun 8: 14063 .

7) Corton JC, Apte U, Anderson SP, Limaye P, Yoon L, Latendresse J, Dunn C, Everitt JI, Voss KA, Swanson C, Kimbrough C, Wong JS, Gill SS, Chandraratna RA, Kwak MK, Kensler TW, Stulnig TM, Steffensen KR, Gustafsson JA, Mehendale HM. 2004. Mimetics of caloric restriction include agonists of lipid-activated nuclear receptors. J Biol Chem 279: 46204-46212.

8) Ingram DK, Zhu M, Mamczarz J, Zou S, Lane MA, Roth GS, de Cabo R. 2006. Calorie restriction mimetics: an emerging research field. Aging Cell 5: 97-108.

9) Spindler SR. 2006. Use of microarray biomarkers to identify longevity therapeutics. Aging Cell 5: 39-50.

10) Chiba T, Komatsu T, Nakayama M, Adachi T, Tamashiro Y, Hayashi H, Yamaza H, Higami Y, Shimokawa I. 2009. Similar metabolic responses to calorie restriction in lean and obese Zucker rats. Mol Cell Endocrinol 309: 17-25.

11) Newman JC, Milman S, Hashmi SK, Austad SN, Kirkland JL, Halter JB, Barzilai N. 2016. Strategies and challenges in clinical trials targeting human aging. J Gerontol A Biol Sci Med Sci 71: 1424-1434.

12) Ito T, Miyazaki N, Schaffer S, Azuma J. 2015. Potential anti-aging role of taurine via proper protein folding: A study from taurine transporter knockout mouse. Adv Exp Med Biol 803: 481-487.

13) Gaull GE. 1989. Taurine in pediatric nutrition: review and update. Pediatrics 83: 433-442.

14) Murakami S. 2015. Role of taurine in the pathogenesis of obesity. Molec Nutr Food Res 59: 1353-1363.

15) Ito T, Hanahata Y, Kine K, Murakami S, Schaffer SW. 2018. Tissue taurine depletion induces profibrotic pat- tern of gene expression and causes aging-related cardiac fibrosis in heart in mice. Biol Pharmaceut Bull 41: 15611566.

16) Ito T, Kimura $Y$, Uozumi $Y$, Takai M, Muraoka S, Matsuda T, Ueki K, Yoshiyama M, Ikawa M, Okabe M, Schaffer SW, Fujio Y, Azuma J. 2008. Taurine depletion caused by knocking out the taurine transporter gene leads to cardiomyopathy with cardiac atrophy. J Mol Cell Cardiol 44: 927-937.

17) Schuller-Levis GB, Park E. 2003. Taurine: new implications for an old amino acid. FEMS Microbiol Lett 226: 195-202.

18) Murakami S. 2017. The physiological and pathophysiological roles of taurine in adipose tissue in relation to obesity. Life Sci 186: 80-86.

19) Shimada K, Jong CJ, Takahashi K, Schaffer SW. 2015. Role of ROS production and turnover in the antioxidant activity of taurine. Adv Exp Med Biol 803: 581-596.

20) Jong CJ, Ito T, Prentice H, Wu JY, Schaffer SW. 2017. Role of mitochondria and endoplasmic reticulum in taurinedeficiency-mediated apoptosis. Nutrients 9: E795.

21) Fukuda N, Yoshitama A, Sugita S, Fujita M, Murakami S. 2011. Dietary taurine reduces hepatic secretion of cholesteryl ester and enhances fatty acid oxidation in rats fed a high-cholesterol diet. J Nutr Sci Vitaminol 57: $144-149$.

22) Folch J, Lees M, Sloane Stanley GH. 1957. A simple method for the isolation and purification of total lipides from animal tissues. J Biol Chem 226: 497-509.

23) Fujii N, Narita T, Okita N, Kobayashi M, Furuta Y, Chujo Y, Sakai M, Yamada A, Takeda K, Konishi T, Sudo Y, Shimokawa I, Higami Y. 2017. Sterol regulatory elementbinding protein-1c orchestrates metabolic remodeling of white adipose tissue by caloric restriction. Aging Cell 16: 508-517.

24) Higami Y, Tsuchiya T, Chiba T, Yamaza H, Muraoka I, Hirose M, Komatsu T, Shimokawa I. 2006. Hepatic gene expression profile of lipid metabolism in rats: Impact of caloric restriction and growth hormone/insulin-like growth factor-1 suppression. J Gerontol A Biol Sci Med Sci 61: 1099-1110.

25) Chiba T, Tamashiro Y, Park D, Kusudo T, Fujie R, Komatsu T, Kim SE, Park S, Hayashi H, Mori R, Yamashita H, Chung HY, Shimokawa I. 2014. A key role for neuropeptide $\mathrm{Y}$ in lifespan extension and cancer suppression via dietary restriction. Sci Rep 4: 4517.

26) Chiba T, Tsuchiya T, Komatsu T, Mori R, Hayashi H, Shimano H, Spindler SR, Shimokawa I. 2010. Development of a bioassay to screen for chemicals mimicking the anti-aging effects of calorie restriction. Biochem Biophys Res Commun 401: 213-218.

27) Yang W, Huang J, Xiao B, Liu Y, Zhu Y, Wang F, Sun S. 2017. Taurine protects mouse spermatocytes from ionizing radiation-induced damage through activation of Nrf2/HO-1 signaling. Cell Physiol Biochem: Int J Exp Cell Physiol Biochem Pharmacol 44: 1629-1639.

28) Tadros MG, Khalifa AE, Abdel-Naim AB, Arafa HM. 2005. Neuroprotective effect of taurine in 3-nitropropionic acid-induced experimental animal model of Huntington's disease phenotype. Pharmacol Biochem Behav 82: 574-582.

29) von Thaler AK, Kamenisch Y, Berneburg M. 2010. The role of ultraviolet radiation in melanomagenesis. Exp Dermatol 19: 81-88. 